\title{
LA PROFESIÓN DOCENTE DESDE UNA MIRADA SISTÉMICA
}

\author{
THE TEACHING PROFESSION \\ FROM A SYSTEMIC PERSPECTIVE
}

Àngels Domingo Roget

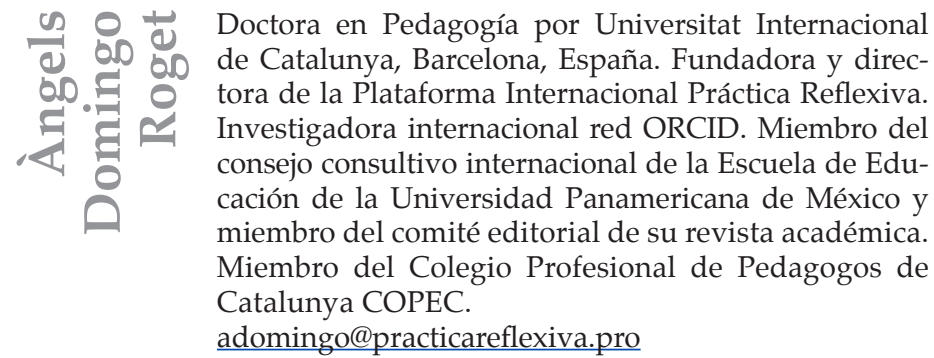

\section{RESUMEN}

Este artículo analiza, desde la perspectiva sistémica, las características del aula como ecosistema vivo y dinámico, y plantea cómo su desafiante gestión por parte del profesor constituye un activador del proceso de la profesionalización docente. La vivencia, la experiencia y algunas características específicas del escenario profesional del docente agregan elementos nuevos para su reflexión y mejora continua.

Desde una pedagogía sistémica, sensible y atenta a los procesos de retroalimentación que se producen en el aula, se invita al docente a reflexionar sobre su práctica y sus experiencias profesionales con el propósito de transformar ese aprendizaje experiencial y reflexivo en fuente de retroalimentación profesional para el mejoramiento continuo de la práctica docente. 
Palabras clave: desarrollo docente, práctica reflexiva, profesión docente, aprendizaje experiencial, formación de profesorado.

\begin{abstract}
This article analyzes from the systemic perspective the characteristics of the classroom as a living and dynamic ecosystem and discusses how it's challenging management, is an activator of the process of teacher professionalization. The experience and some specific characteristics of the teachers' professional scenario add new elements for reflection and continuous improvement.

From a systemic pedagogy, always sensitive and attentive to feedback processes occurring in the classroom, the teacher is prompted to reflect on her practice and experience with the purpose of transforming the experiential and reflective learning into a source of professional feedback for continuous improvement of the teaching practice.
\end{abstract}

Keywords: Teacher Development, Reflective Practice, Teaching Profession, Experiential Learning, Teacher Training.

\title{
LA EXPERIENCIA EN EL MARCO DEL DESARROLLO PROFESIONAL
}

Desde la perspectiva pedagógica y existencial, podría afirmarse que uno de los momentos más desafiantes en la vida profesional de un docente novel es su inicio en la profesión: cuando llega al escenario profesional. Es cuando comprueba la enorme diferencia del contexto académico previo y el escenario real, y experimenta la distinción entre el mundo académico vivido y el mundo profesional. Comienza para el docente, para ya no terminar nunca, la vivencia denominada disonancia cognitiva o conflicto cognitivo, que enfrenta el conocimiento teórico con las situaciones prácticas del aula que le corresponde abordar. Es una etapa retadora que todo docente ha vivido y del que fácilmente podríamos recordar 
episodios inolvidables por el impacto emocional que generaron en su momento. Todo el saber académico aprendido durante la formación inicial, se enfrenta a la práctica. En esta situación, el docente novel afronta una nueva asignatura no curricular: aprendizaje experiencial (Kolb, 1984).

El aprendizaje experiencial es aquel que procede de la experiencia y que potencia la adquisición de las competencias específicas de la profesión docente. Podemos describir el aprendizaje experiencial como un trabajo reflexivo sobre lo vivido, una transformación de la experiencia en conciencia dentro de un proceso de autoafirmación que tiene lugar en todas las situaciones de toma de decisiones y de resolución de problemas en la vida cotidiana (Domingo, A., 2019: 40).

Comienza entonces para el docente un itinerario profesionalizador que recorrerá de forma expectante y a la escucha de las sabias lecciones de la experiencia. El aprendizaje experiencial es el que proporciona un conocimiento directo de la realidad educativa y nutre al docente de elementos para una reflexión orientada a la intervención en los procesos de enseñanza-aprendizaje y en la participación en la dinámica del aula.

En este mismo sentido, el aprendizaje puede ser descrito como el resultado de la reflexión sobre la experiencia que encauza a una acción intencional de cara a comprobar las hipótesis que surgen de dicha reflexión. Esta acción lleva por su parte a una experiencia más profunda y a una nueva reflexión, de forma que el aprendizaje experiencial puede ser concebido como un ciclo continuo o espiral. Por esta razón, resultan en la práctica inseparables las nociones de aprendizaje experiencial (que procede de la experiencia) y el aprendizaje reflexivo (que requiere reflexión).

Para que surga el aprendizaje experiencial se precisan básicamente tres elementos distintos que estén a su vez interactuando. Esos elementos básicos son: experiencia, reflexión y aprendizaje. En la siguiente figura se muestra esos elementos básicos y la relación entre sí. 
Figura 1. Aprendizaje experiencial: elementos y estructura.

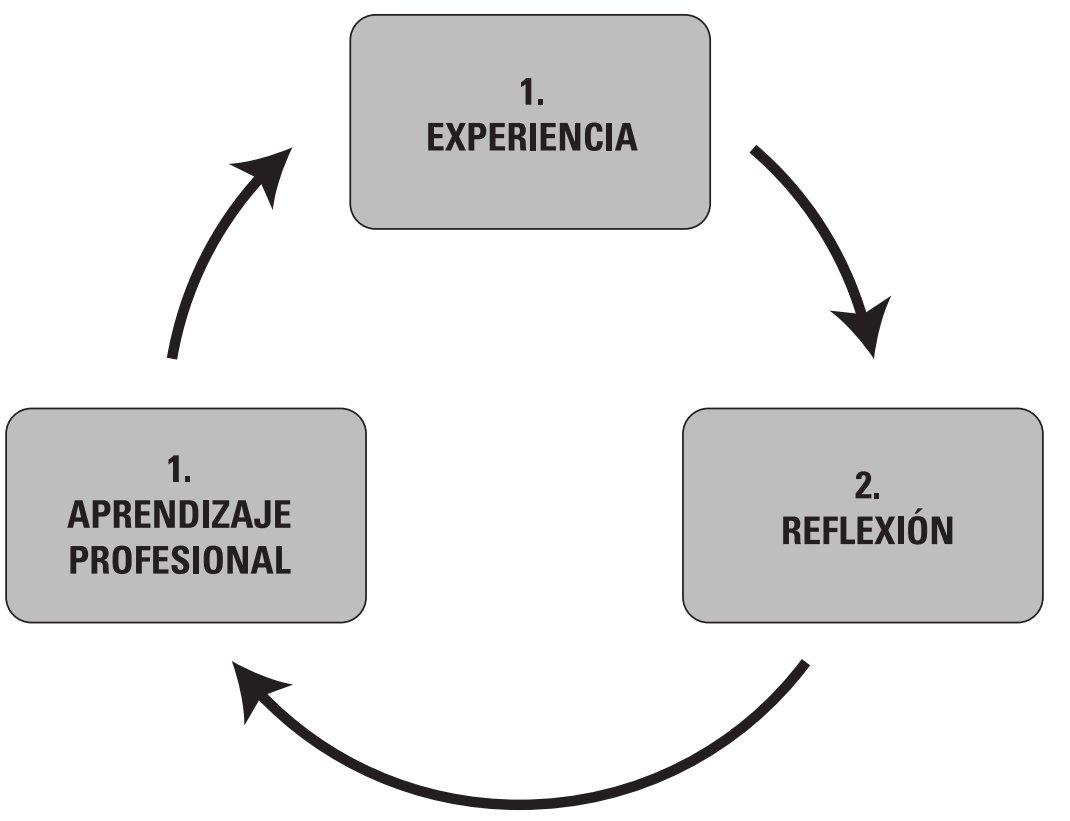

Fuente: Elaboración propia (2013).

Conviene tener presente que la experiencia, que es el elemento clave, puede resultar en sí misma insuficiente como base para el desarrollo profesional. Precisamente cuando el aprendiz actúa en este proceso didáctico como un participante activo, comprometido con su propio aprendizaje, y mantiene una interacción continua entre la acción y la reflexión, es cuando podemos hablar propiamente de aprendizaje experiencial.

Para el docente novel, la experiencia que vive en las aulas constituye un activador de desarrollo profesional que le induce a desarrollar 
habilidades docentes y el análisis crítico-reflexivo sobre su práctica. Este fenómeno interno y subjetivo que se describe es muy destacado en el caso del profesor novel; sin embargo, sabemos que se transforma posteriormente en un dispositivo profesional que, de forma estable, retroalimenta la mejora del desempeño docente, también de los docentes expertos que destacan por la calidad de su docencia. Precisamente a más experto es un profesor, mayor capacidad de aprender de la experiencia reflexionada.

Sentadas estas bases que se sustentan en los resultados de recientes investigaciones, nos interesa prestar atención a dos elementos configuradores de la identidad docente que están estrechamente vinculados con la formación docente práctica: en primer lugar, la fuerza de un contexto educativo en el desarrollo profesional y en segundo lugar, la necesidad de la frecuente toma de decisiones en la profesión docente en situaciones de inmediatez e incertidumbre. Esas dos realidades que se destacan son elementos característicos de la profesión y confluyen inexorablemente en la práctica educativa al ejercer una fuerte presión sobre el docente e incidir en su intervención educativa.

\section{EL CONTEXTO EDUCATIVO}

Toda profesión se identifica por su vinculación a una serie de cuestiones y problemas que le son propios. Algunas profesiones tienen muy bien delimitados esos ámbitos de actuación mientras que en otras profesiones están menos delimitados. Además, las dificultades de delimitación de la actuación profesional plantean unas dificultades añadidas, como son la misma complejidad del concepto de educación y la dificultad de que la solución a los problemas educativos que se presentan en el aula no depende exclusivamente de la actuación profesional. Las situación humana que plantea el aula no se puede prever con exactitud por la riqueza de las variables que intervienen en la situación. Por ello, salvando las distancias, lo considero como un ecosistema vivo y dinámico.

Los modelos ecológico-contextuales estudian el aula como una realidad física y psicosocial en que se produce un complejo y dinámico 
entramado de variables que interactúan de formas diversas y que hacen de cada aula una realidad singular. Superados los planteamientos positivistas radicales, los avances de investigación no se inclinan hacia la búsqueda de una teoría educativa que decida por los docentes, sino que aportan teorías que orienten a estos para asegurar que sus decisiones tengan buen fundamento.

El alcance con que el contexto influye en la enseñanza - y determina qué enfoques de la enseñanza serán eficaces- es un factor de reconocimiento creciente, reconocido en la investigación sobre la enseñanza, en la formación del profesorado y en la evaluación de la enseñanza (Darling-Hammond y Snyder, 2000:524).

En el caso de la educación, el contexto puede ser muy distinto de una situación a otra, puesto que los agentes y las situaciones educativas de la sociedad lo son. Para nuestro análisis nos interesa solamente el contexto de la enseñanza formal.

La expresión contexto, originaria de las ciencias de la comunicación y de la informática, así como de la semiología y la lingüística, podría entenderse en el ámbito pedagógico como el conjunto de circunstancias bajo las cuales se produce un acto cognitivo en una situación educativa, tales como: lugar, tiempo, emisor, receptor, condiciones, entorno, ambiente, etcétera. Son precisamente esas circunstancias concretas las que contextualizan el hecho educativo. Estos contextos no son genéricos ni generalizables, ni pueden ser estudiados de forma teórica y sistemática; podemos afirmar que los contextos nunca son generales, por su misma naturaleza, y son extraordinariamente concretos.

Desde una perspectiva socio-constructivista, más centrada en la dimensión subjetiva y social de la persona, se entiende por contexto el fenómeno mental por el cual la persona que habla o escucha aprovecha cada acto de comunicación para entender la información y lo que los demás le comunican (Mercer, 2000). Este proceso consiste básicamente en vincular lo nuevo con lo conocido, ofreciendo una base común para todos los interlocutores. En consecuencia los interlocutores, juntamente, crean el contexto. 
El conocimiento de la diversidad de los contextos educativos constituye uno de los aprendizajes específicos de los profesores y maestros en activo o en servicio.

Tal como apunta Imbernón (2007: 39)

Hay acuerdo en considerar que es difícil generalizar situaciones de enseñanza, ya que la función docente no se enfrenta a meros problemas, sino a situaciones problemáticas contextualizadas.

Partiendo de la información que emana del contexto, el docente analiza el modo de pensar y aprender de su alumnado. Las cuestiones sobre comportamiento y disciplina de los alumnos, así como su motivación e interés por aprender, suele suscitar en el profesor replanteamientos en sus posturas pedagógicas, e involucrado en una inesperada dinámica en el aula, fácilmente adopta nuevos principios metodológicos como respuesta a situaciones reales. En esta situación experiencial, el docente se siente inducido a armonizar un difícil, y a la vez fecundo, diálogo entre las tres interpretaciones pedagógicas que le presentan las situaciones reales: la pedagogía general, basada en grandes principios deductivos; la pedagogía experimental de carácter inductivo; y la pedagogía en situación, sustentada en un enfoque etnográfico y contextual. En el docente, este diálogo pluridisciplinar subjetivo, refuerza su profesionalidad por medio de recursos y habilidades intelectuales que le conducen a articular, vertebrar, dialogar, confrontar y elaborar soluciones contextualizadas.

Basil Bernstein (1977), sociólogo inglés, de la segunda mitad del siglo XX, planteaba que la potencialidad del conocimiento depende en buena parte de los contextos en los cuales se desarrolla. Distinguía tres contextos: contexto de producción, contexto de aplicación y contexto de reproducción de conocimiento.

a) En el contexto de producción de conocimientos se busca el conocimiento por sí mismo. Lleva aparejada la motivación de aprender porque la persona —en nuestro caso, el docente-, al estar produciendo conocimiento, se sumerge en la aventura del conocimiento, 
en la aventura de descubrir, de contrastar, de experimentar, en la aventura de ir recreando el conocimiento permanentemente $y$, por tanto, ese contexto va ligado al proceso de motivación personal.

b) En el contexto de aplicación ocurre algo similar al contexto anterior: también la persona en esa situación está motivada para aprender porque el conocimiento que utiliza resuelve problemas, modifica realidades, transforma situaciones, aunque sea un conocimiento no producido por el mismo aprendiz, sí es un conocimiento comprendido y aplicado por el propio sujeto a la transformación de la realidad.

c) En el contexto de reproducción del conocimiento es donde resulta muy difícil encontrar motivación intrínseca. En esta situación, el contexto de intercambio de conocimiento se convierte exclusivamente en reproducción del conocimiento sin acceso a la producción de conocimiento ni acceso a su aplicación. La repetición mecánica no provoca motivación por el aprender ni adquisición útil de un conocimiento que nos sirve para entender mejor la realidad o para transformar la realidad en la que se está interviniendo.

Las aulas y su realidad contextual activan en el docente su profesionalización por medio de la producción y aplicación del conocimiento a cada contexto distinto, y evitando ejercitarse en la reproducción mecánica.

\section{UN ENFOQUE SISTÉMICO}

En la profesión docente, la necesidad de contextualización ha ido adquiriendo mayor fuerza por la influencia de la perspectiva sistémica en los fenómenos educativos. La perspectiva sistémica, procedente del ámbito de la biología, utiliza la imagen de sistema para una comprensión más completa y profunda de la educación y de la didáctica. Precisamente el aula, el escenario profesional del docente, es una realidad de carácter sistémico. Por esta razón algunas reflexiones sobre la teoría de sistemas y la perspectiva sistémica pueden ilustrar en este punto. 
Traveset (2007) lo explica así:

Un sistema se define como un conjunto de elementos en interacción entre ellos y de forma conjunta con el entorno. Cada elemento se puede estudiar de manera aislada, pero sólo adquiere significado en la medida que es considerado parte integrante de un todo; por tanto, cualquier estudio de un elemento aislado es parcial, y cualquier elemento puede verse como un sistema que, al mismo tiempo, forma parte de un sistema mayor. Esto implica configurar el universo como una arquitectura de sistemas en interacción y con unos órdenes jerárquicos (Traveset, 2007:18).

En la línea de las consideraciones, la pedagogía sistémica se considera una nueva forma de mirar que implica cambios profundos y cuyo enfoque trata de crear las condiciones idóneas para que el aula, la escuela, la universidad, etcétera, se perciban como una realidad abierta e interconectada de manera permanente con su entorno. Esta percepción sistémica facilita el reconocimiento de un flujo continuo de energía del propio sistema. Los conceptos de retroalimentación - el llamado feedback - y auto organización permiten entender el momento presente en cada sistema y en el aula como el resultado de una dinámica de cambio evolutivo en el que se integra a la vez el azar y la necesidad, que hace posible que surjan nuevas situaciones inicialmente no previstas. La pedagogía sistémica y su visión de la educación obliga al sistema a conciliar el orden con el desorden, y a reconocer y gestionar el flujo continuo de energía del propio sistema, y la emergencia de lo nuevo y lo creativo.

En este sentido, el estudio del aula se puede abordar como una unidad, pero al mismo tiempo cada uno de sus elementos posee unas características específicas que reflejan su interrelación con el resto. El pensamiento sistémico se diferencia del pensamiento lineal-lógico, basado en relaciones causa-efecto y en el dualismo, lo que supone fijar la mirada en el problema, atomizar, reducir el problema a una parte y aplicar a esta la solución dejando de ver el todo y su interacción con el entorno. Esta visión aún continúa vigente en muchos aspectos del sistema educativo y en muchas áreas de la ciencia: la separación de las disciplinas; no tener en cuenta el contexto; el trabajo individual de los 
alumnos; no tener presente la dinámica de los grupos y las fuerzas en interacción; primar los conocimientos muy académicos y desvinculados de la condición humana y que disocian lo racional de lo emocional, etcétera.

El enfoque sistémico centra su visión en la conectividad relacional. Lo nuclear de los hechos que suceden en el aula no es el suceso en sí mismo, sino la acción recíproca de los distintos elementos que componen la situación educativa o didáctica.

Si el docente observa de forma sistémica el aula, percibe una serie de fuerzas poco tangibles, pero de gran densidad, como la que presentan los alumnos individualmente, la fuerza de su historia personal, de su familia, la fuerza del grupo como tal, sus conocimientos previos, etcétera, y comprende que estas fuerzas pueden intervenir con relevancia en el proceso del aprendizaje.

El orden es uno de los elementos básicos para que los sistemas y organizaciones humanas —escuela, familia, empresa- funcionen. Aunque los sistemas sean distintos, el orden resulta un valor universal e imprescindible para todos ellos y les garantiza la funcionalidad. El desorden también es propio de todos los sistemas, y precisamente la perspectiva sistémica pretende ampliar la mirada para mostrar los órdenes que subyacen en las dinámicas implicadas en los espacios de aprendizaje: familiar, escolar, laboral, social, cultural. Identificado el desorden su objetivo es reconstruirlo y restablecer la funcionalidad del sistema. La interpretación sistémica del hecho educativo pretende primordialmente descubrir el orden natural e identificar los desórdenes y colocar en su lugar correspondiente los distintos elementos que intervienen en la situación educativa (docente, alumnos, familia, escuela, etcétera) para integrar, equilibrada y saludablemente, todas esas realidades diversas en la unidad de cada alumno único y singular. Se trata de una pedagogía de corte fenomenológico en cuanto pretende comprender cómo vive el alumno, su realidad desde el punto de vista subjetivo, y procura determinar el sentido dado a los fenómenos, descubrir el significado y la forma, cómo las personas describen su experiencia subjetiva en situaciones concretas. La orientación fenomenológica 
intenta descubrir todo aquello que aparece como pertinente y significativo en las percepciones, sentimientos y acciones de los agentes educativos.

Desde esta perspectiva sistémica, que constituye una preparación teórico-práctica interactiva amplia, el docente gestiona prudente y respetuosamente los elementos contextuales que inciden en el aula y en cada uno de los alumnos, y será capaz de encontrar soluciones más eficaces, más amplias y profundas, y podrá abordar mejor el complejo proceso educativo en los espacios de aprendizaje. Este enfoque pedagógico-sistémico permitirá contemplar e integrar la realidad educativa como un todo, vinculado a los sistemas familiares, sociales, culturales e históricos, que son los que desde su base están influyendo y repercutiendo en todo el proceso de enseñanza-aprendizaje.

Los docentes solo pueden conocer la relevancia del contexto en los procesos y fenómenos educativos desde una perspectiva práctica. La verdadera contextualización de su tarea únicamente se hace realidad cuando permanecen y trabajan en el aula in situ, interaccionando con los alumnos y su contexto. Exclusivamente en esas condiciones, el docente puede conocer y abordar las coordenadas contextuales de su trabajo.

\section{INMEDIATEZ Y TOMA DE DECISIONES}

Indaguemos ahora en la otra característica del aula - ya comentada- que incide directamente en la configuración de la identidad profesional del docente y que actúa de forma relevante en su proceso de profesionalización.

Tras muchos estudios, los expertos sobre organizaciones humanas reconocen la imposibilidad de entenderlas sin apoyarse en un modelo básico del hombre y, especialmente, en su forma de actuar y tomar decisiones. La gestión de las organizaciones o microorganizaciones - como puede ser el aula escolar- se apoyan en el comportamiento humano. 
Durante la etapa de formación inicial un futuro docente avanza en su formación pedagógica, didáctica, psicopedagógica, decidiendo, de un modo más o menos consciente, sus opciones pedagógicas personales ante las diversas posibilidades que presentan las distintas materias de su programa de estudios. En esa situación formativa, únicamente toma decisiones al proyectarse en su futuro profesional como docente y va alimentando la intención o deseo de lograr ser un maestro con un perfil determinado y que incluye, además del bagaje pedagógico y didáctico, otros muchos elementos que configuran su personalidad. En ese período, el futuro maestro construye progresivamente su propio estilo y maneras docentes; toma determinaciones teóricas hacia las que se siente más inclinado y opta por decisiones acordes con su propia sensibilidad educativa, y sus valores personales y humanos. En ese itinerario pedagógico exclusivamente personal que cada uno traza para su futura tarea docente, influyen decisivamente los modelos docentes de los profesores que les preparan y que, siendo muy heterogéneos, les sitúa ante diferentes opciones metodológicas, didácticas, sociales, intelectuales, evaluativas, etcétera. Todo este proceso descrito no siempre es consciente, pero sí es siempre un proceso lento, interior, sereno, abierto, dinámico y revisable por parte del futuro docente con motivo de nuevas vivencias o influencias. El clima del proceso es sosegado, reversible, sin riesgo ni consecuencias en los demás.

Todo eso cambia si analizamos el aula como espacio de desempeño del docente en servicio: el contexto y las coordenadas reales ejercen presión y las decisiones e intervenciones ahora sí tienen consecuencias en sus alumnos.

Para reflexionar sobre esta nota de la inmediatez y la toma de decisiones del docente cuando enseña, pueden ilustrarnos los análisis que realizan los expertos en organizaciones humanas que estudian también la toma de decisiones en situaciones de inmediatez o de incertidumbre, aunque en otros contextos. Estos parten de la base de que definir o describir el comportamiento de un modo completo es habitualmente problemático. Aun existiendo estructuras formales que contribuyen a sistematizar el proceso para decidir, conviene tener presente que, en los ámbitos educativos, hay que contar con un plus de actividades de los diferentes agentes implicados que no pueden ser explícitamente definidas 
y que, sin embargo, son relevantes para el logro de resultados. Este tipo de actividades suelen denominarse comportamientos implícitos, entendidos como formas de comportamiento no formalizadas - es decir, no abarcables desde la estructura formal-, que rigen y determinan las decisiones de los individuos y, en consecuencia, no pueden ser objeto de previsión ni de control. Esto provoca que el docente, que ha planificado sus tareas, ante las inevitables contingencias imprevistas en el aula, sea presionado por la situación a buscar una respuesta no preestablecida. Los comportamientos implícitos de los alumnos, las circunstancias que se imponen, junto a las interacciones que se producen, generan en el docente situaciones de incertidumbre: no solo habrá de decidir entre alternativas posibles sino que, en ocasiones, el problema que ha de resolver en el aula cambia y es distinto del previsto, puesto que cambia su naturaleza y sus variables integrantes (Bastons, 2000). Este mismo autor nos plantea, con realismo, la necesidad de una constante toma de decisiones en algunas profesiones - entre las que se encuentra la docente-y plantea la necesidad del aprendizaje en la toma de decisiones en estas y otras organizaciones:

No se trata en este caso de un cambio de proceso de elección de alternativas, sino de un cambio de las «premisas» —en la estructura-de la decisión. Esto genera una incertidumbre de nivel diferente. En estos casos no se trata de una incertidumbre intrínseca a la elección, sino de una incertidumbre relativa a la estructuración de los problemas de decisión: la modificación de la capacidad operativa del agente. [...] Se trata, pues, de cambios producidos en niveles diferentes de decisión. Unos se refieren a la elección de un agente; otros, a la estructuración de sus problemas de decisión. Se está aludiendo aquí a un fenómeno común en lo que se denominan problemas mal estructurados (Selva, 1993): su naturaleza, sus variables integrantes, se modifican de forma paralela al proceso desarrollado para resolverlos. Una vez que se ha solucionado el problema no queda permanentemente resuelto: nace otro problema. La solución de un problema genera un problema de decisión nuevo, que a su vez requiere nuevas soluciones (Bastons, 2000:132).

En esas situaciones cargadas de incertidumbre, las decisiones del docente puede parecen en apariencia espontáneas, aunque no sea 
así. La incertidumbre lleva al docente, formado profesionalmente, a adoptar una actitud más flexible en las formas de organización personales y escolares, y le conduce a mantener una actitud abierta ante los problemas a resolver. Con frecuencia se ve obligado en plena actividad docente a redefinir y reestructurar de nuevo el problema que ha de resolver. Y aunque la percepción externa parezca aparente espontaneidad en la respuesta a los problemas, hay que resaltar que el profesor reestructura y decide de nuevo siguiendo un proceso dinámico, rápido y holístico, que tiende a poner en el centro la dimensión personal de las situaciones, priorizando racionalmente sus valores.

Las situaciones que vive el docente en su quehacer diario le relacionan con estudiantes de diversas características, tipologías y procedencias heterogéneas desde el punto de vista cultural, social, familiar, etcétera. Percibe con rapidez la complejidad, el dinamismo, la interacción, la responsabilidad de la situación, y las presiones humanas y normativas. Percibe que se mueve en una situación de carácter sistémico y su percepción vivencial del aula es la de un espacio psicosocial de comunicación e intercambios (Doyle, 1985, Pérez Gómez, 1989) en los que los comportamientos de las personas son una respuesta de adaptación contextual global, o un sistema vivo donde sus elementos se definen en función del intercambio y donde el sistema va tomando forma a partir de la participación activa de todas las personas implicadas en los procesos de enseñanza-aprendizaje. Este clima de intercambios constantes son los que, a su vez, generan nuevos roles y patrones de actuación, tanto individuales como grupales. En el aula, el profesor no dispone de tiempo para meditar y su reflexión rápida - y durante la acción- es un medio imprescindible para seguir adelante y guiar el siguiente paso a dar; para decidir el camino que debe seguir, interrumpir o no una charla, empezar o no con un nuevo capítulo, aceptar o no una excusa, intervenir ante la actuación de un niño indisciplinado, cómo responder a una pregunta inesperada, etcétera. Mentalmente, el practicante escasamente puede deliberar y sopesar la inmediatez de las situaciones que le exigen actuar. En esas condiciones apenas toma decisiones explícitas y formales, habitualmente toma muchas $\mathrm{mi}$ crodecisiones (Eggleston, 1989). En la siguiente figura se presenta, 
esquemáticamente, el proceso reflexivo subjetivo del docente para la toma de microdecisiones orientadas a la resolución de situaciones en el aula.

Figura 2. Proceso para la toma de microdecisiones.

\section{Actividad reflexiva del docente}

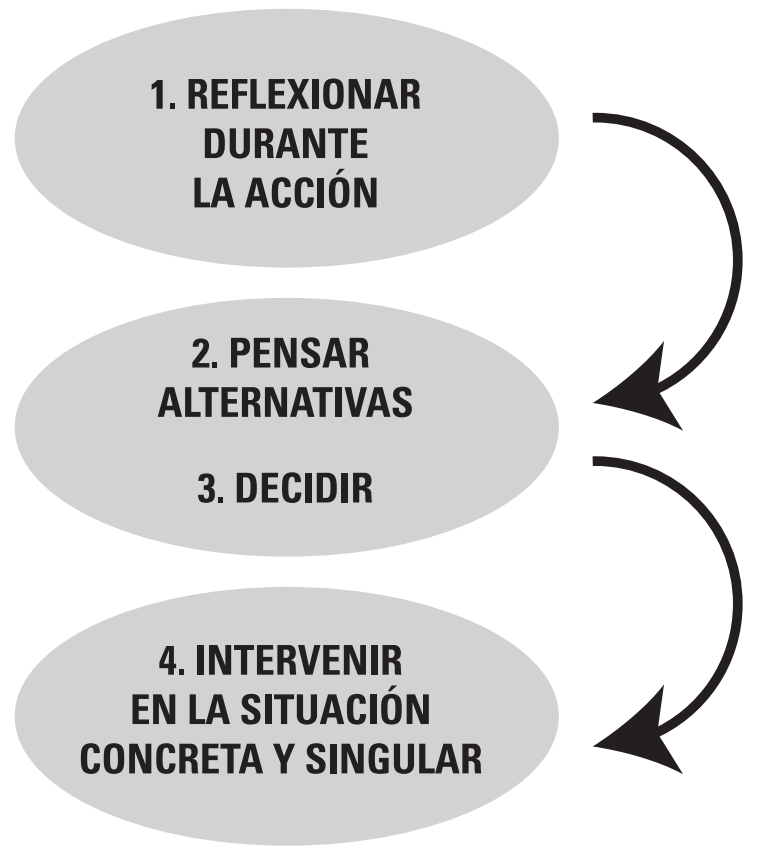

Fuente: Elaboración propia.

Cuando el docente toma decisiones, casi de un modo inconsciente y sin formalizarlo, adopta actitudes de compromiso con las situaciones de los alumnos y se sirve de metodologías de corte cualitativo basadas en la observación, la indagación, el análisis situacional del aula y del contexto en su conjunto. Para comprender mejor 
los escenarios individuales y grupales se sirve de procesos asequibles de triangulación, y de forma natural busca experimentar en la acción. Y para todo ello precisa tomar muchas decisiones para diagnosticar, actuar, intervenir, observar, etcétera. Sin embargo, para realizarlo, el docente no puede detenerse a realizar una reflexión teórica y distante de la situación que vivencia con los estudiantes. La inmediatez de la situación le exige una reflexión inseparable de la acción, a la que Schön llama «reflexión en la acción». Así lo expone Schön (1987:73):

El estudio de la reflexión desde la acción tiene una importancia clave. El dilema del rigor o la relevancia puede ser resuelto si podemos desarrollar una epistemología de la práctica que sitúe la resolución técnica del problema dentro del contexto más amplio de una indagación reflexiva, muestre cómo la reflexión desde la acción puede ser rigurosa por propio derecho, y vincule el arte de la práctica, en la incertidumbre y el carácter único, con el arte de la investigación del científico. Podemos de este modo incrementar la legitimidad de la reflexión desde la acción y alentar su más amplia, profunda y rigurosa utilización.

Para referirse a esa capacidad para adaptarse al contexto cambiante, incierto y momentáneo que caracteriza el aula, Doyle (1985) la denominó competencia situacional y que en estos últimos años, tras recientes investigaciones internacionales, la hemos denominado competencia reflexiva (Domingo A. y Gómez V., 2014:27): «La competencia reflexiva es la capacidad habilidad de efectuar tareas o hacer frente a situaciones diversas de forma eficaz en un contexto determinado mediante la movilización simultánea de actitudes, habilidades y conocimientos».

En esta situación, el profesor deja de ser un técnico que ejecuta destrezas estereotipadas y adquiridas fuera de contexto, y se convierte en un profesional activo, reflexivo, que emite juicios y toma decisiones. Clark (1989) considera al docente como un constructivista que continuamente va elaborando estrategias de actuación a partir de procesos de observación, análisis y reflexión en el contexto en que actúa. De este modo el acto didáctico se perfila como algo momentáneo, situacional, irrepetible, abierto, cargado de inmediatez. Para resolver con éxito las tareas propuestas y otras estrategias cognitivas y afectivas, el estudiante en 
prácticas ha de tomar decisiones permanentemente, puesto que así lo exige su entorno dinámico, vivo y procesual. Habitualmente esas decisiones son las que conducen a la llamada intervención pedagógica, expresión general que resume todas aquellas medidas, decisiones y microdecisiones (Eggleston, J., 1989) que adopta el docente en relación con la educación en el aula.

El docente no puede limitarse estrictamente a «enseñar», pues es un verdadero profesional que orienta, planifica, socializa, dinamiza, organiza, valora, selecciona y elabora recursos, evalúa, etcétera. Su tarea requiere un perfil plenamente profesional, capaz de realizar unas determinadas tareas, aplicar conocimientos científicos y técnicos para resolver los problemas que el aula y los alumnos plantean, individual o grupalmente. Y resolver positivamente problemas significa que ese profesional de la educación los identifica adecuadamente, los estructura de forma correcta, toma decisiones acertadas, y posteriormente —aunque el proceso citado sea muy rápido en el tiempo-, interviene pedagógicamente de forma eficaz y logrando los resultados previstos.

Otra muestra de la profesionalidad que se le reconoce al docente es el respeto a su autonomía de acción. La naturaleza de su actividad educativa lo exige, puesto que los conocimientos y habilidades no son únicos y cabe optar en cada caso por la mejor estrategia de acción en función de las circunstancias. Tampoco se puede olvidar que cualquier estrategia metodológica está condicionada por la personalidad de quien la aplica, de modo que su efectividad dependerá de las actitudes y habilidades del docente. Por otra parte, la normativa legal señala directrices básicas y mínimos curriculares que cabe adaptar a cada situación concreta de acuerdo con los criterios profesionales apropiados, lo cual, en definitiva, requiere la libertad de acción. Acertadamente, Sarramona (2002) establece como límite de esa libertad de acción propia del profesional, la dimensión moral de la acción educativa.

[... La libertad de acción tendrá como límite la propia deontología profesional y el estado del desarrollo científico y técnico de la profesión, además de la experiencia y reflexión personales. Esto significa que en nombre de la libertad de acción no se puede desconocer el patrimonio científico de la profesión, ni se pueden adoptar decisiones que solo se 
amparan en la tradición o la rutina. La toma de decisión en autonomía ha de servir para potenciar la capacidad reflexiva e innovadora del profesorado y nunca una excusa para no desarrollarse profesionalmente, ni para romper la cohesión institucional, marcada por los proyectos educativos del centro.

La deontología aplicada a la educación tiene otras implicaciones que no pueden soslayarse. El educador no puede separar lo que es de lo que hace, y los criterios morales aplicados a la actividad educativa repercuten directamente sobre su personalidad, puesto que el ejemplo sigue siendo pieza fundamental en la tarea de educar.

El educador difícilmente podrá educar en valores que no comparta, de modo que dedicarse profesionalmente a educar supone un compromiso personal con un proyecto moral (Sarramona, 2002:188-189).

Si en términos generales afirmamos que la educación conlleva una considerable dimensión moral, la ética juega un importante papel en la toma de decisiones. Precisamente el contexto humano del aula y la educación afectan éticamente al docente, propiciándole un conflicto de valores que ha de resolver con ética profesional. Bastons (2000) amplía el concepto de «sistema abierto incorporando la dimensión valorativa y ética de las decisiones»:

La consideración de la organización como sistema abierto, típica de los enfoques ambientales, permite hacerse cargo de la inestabilidad del entorno, incorporando el aprendizaje instrumental y el ambiente como mecanismo de control. Pero resulta insuficiente para optimizar la estructuración de las decisiones cuando la evaluación de los resultados también resulta inestimable. Hace falta que la organización sea un sistema abierto, pero no solo hacia los resultados externos o las reacciones del entorno, sino también hacia las satisfacciones y las preferencias por el logro de esos resultados. No es suficiente, pues, con la capacidad de adaptación. Es necesario también optimizar la capacidad de valoración. De eso se ocupa la ética (Bastons, 2000:166).

Para concluir este análisis del escenario profesional que incide directamente en la configuración de la identidad docente, se apuntan algunos los postulados que aporta la perspectiva sistémica sobre la 
profesión docente. Se integran en su enumeración resultados de la investigación sobre Desarrollo Docente a través de la Práctica Reflexiva (Domingo A., 2013).

En buena medida estos postulados constituyen retos y nos ofrecen una prospectiva para la acción y la investigación de las próximas décadas.

- La perspectiva sistémica de la profesión docente otorga relevancia al aprendizaje experiencial y reflexivo como elemento activador de la profesionalización docente, y como sistema de mejora permanente de la práctica docente.

- El profesor precisa dos competencias docentes que solo puede adquirir y desarrollar a través de su propia práctica reflexiva: saber contextualizar y saber tomar decisiones durante la acción en situaciones de inmediatez e incertidumbre.

- La profesionalidad del docente se evidencia y pone de manifiesto cuando, en situaciones de cambio de premisas previstas y de inmediatez de nuevas situaciones en el aula, es capaz de reestructurar de nuevo el problema y tomar decisiones.

- La profesionalidad del docente exige que este sea capaz de reflexionar en plena acción sin necesidad de interrumpirla y tomar decisiones, independientemente de la escasez de tiempo o de la inmediatez de la situación.

- Conocidas las características específicas del escenario profesional, el docente precisa disponer de una metodología reflexiva ágil, operativa y lo más holística posible, que tenga en consideración el máximo de elementos y variables posibles.

- Una formación inicial del docente más holística, más interdisciplinar y transdiciplinar, y menos lineal y fragmentaria, facilitará que el docente esté mejor preparado y sea más competente en la toma de decisiones y la resolución de problemas en el aula, lugar de muchas $\mathrm{y}$ diversas interacciones. 
- La formación inicial debe preparar a los nuevos docentes en la experimentación de modelos y metodologías de práctica reflexiva con el fin de que puedan ejercitarse en ella durante su desempeño profesional, y así entrar en el ciclo de la mejora continua de su propia práctica.

\section{REFERENCIAS}

Bastons, M. (2000). La toma de decisiones en la organización. Barcelona: Ariel.

Clark, C. (1989). «Proceso de pensamiento de los docentes», en WITTROCK, M. C. (Ed). La investigación de la enseñanza. Vol III. Barcelona: Paidós.

Darling-Hammond, L. y Snyder, J. "Authentic Assessment of Teaching in Context», en Teaching and Teacher Education, 16 (2000), 523-545.

Domingo, À. (2019). «El aprendizaje experiencial en la profesión docente» en A. Domingo et al. (2019). La Formación del Docente y la Práctica Reflexiva. México: CINADE.

Domingo, À. y Gómez, V. (2014). La Práctica Reflexiva. Bases, modelos e instrumentos. Madrid: Narcea.

Domingo, À. (2013). Práctica reflexiva para docentes. De la reflexión ocasional a la reflexión metodológica. Saarbrücken: Publicia Ed.

Doyle, W. (1985). «Learning to teach: An emerging direction in resseach on preservice teacher education", en Journal of teacher education $n^{\circ} 36$, pp. 31-32.

Eggleston, J. (1989). Teacher decision making in the classroom. London: Routledge and Kegan.

Imbernon, F. (2007). La formación y el desarrollo profesional del profesorado. Hacia una nueva cultura profesional. Barcelona: Graó. 
Kolb, D. (1984). Experiential learning. Englewoods Cliffs, Prentice Hall. Mercer, N. (2000). Lenguaje y pensamiento. Barcelona: Paidós.

Sarramona, J. (2002). «La acción educativa» en Colom, A.; Bernabeu, J.L. y Domínguez, E. en Teorías e instituciones contemporáneas de la educación. Barcelona: Ariel Educación.

Schön, D.A. (1987). La formación de profesionales reflexivos. Hacia un nuevo diseño de la enseñanza y el aprendizaje de las profesiones. Barcelona: Paidós.

Traveset, M. (2007). La pedagogía sistémica. Fundamentos y práctica. Barcelona: Graó. 